15. Lewis JS, Jorgensen JH. Inducible clindamycin resistance in staphylococci: should clinicians and microbiologists be concerned? Clin Infect Dis. 2005;40:280-285.

16. Rao GG. Should clindamycin be used in treatment of patients with infections caused by erythromycin resistant staphylococci? J Antimicrob Chemother. 2000;45:715.
17. Levin TP, et al. Potential clindamycin resistance in clindamycin susceptible, erythromycin resistant Staphylococcus aureus: report a clinical failure. Antimicrob Agents Chemother. 2005;49:1222-1224.

18. Siberry GK, Tekle T, Carroll K, Dick J. Failure of clindamycin treatment of methicillin resistant Staphylococcus aureus expressing inducible clindamycin resistance in vitro. Clin Infect Dis. 2003;37:1257-1260.

\section{Journal Scan}

Sui G.Y, Liu G.C, Liu G.Y, et al. Is sunlight exposure a risk factor for age related macular degeneration? A systematic review and meta-analysis. $\mathrm{Br} \mathrm{J}$ Ophthalmol. 2013;97: 389-394.

Age related macular degeneration (AMD) is a leading cause of blindness worldwide in the age group above 50 years. Two types have been described, the dry type which causes slow but irreversible blindness in advanced stages accounting for $90 \%$ of the total AMD and the wet type which causes rapid loss of vision and constitutes the remaining $10 \%$. Presently the wet type is managed with intra-vitreal injection of anti-Vascular Endothelial Growth Factors (VEGF) whereas there is no curative treatment for advanced dry AMD. Current strategies are aimed at prevention of dry AMD. The risk factors that have been identified include age, gender, genetic factors, smoking, cardiovascular disease, and iris colour and sunlight exposure. However the data on sunlight exposure has been varied and rather controversial. In this article the authors have done a systematic review and meta-analysis to assess the association between sunlight exposure and AMD with the hypothesis that sunlight exposure is indeed a risk factor for AMD and that heterogeneity between studies is due to study-specific covariates, including latitude and gross domestic product (GDP) per capita. An electronic search was performed of PubMed, Web of Science and CNKI, which was supplemented by hand searching. The selection of studies, data abstraction and quality assessment were performed independently by three reviewers. Epidemiological studies that met both of the following items were selected: the studies concerning the association between AMD and sunlight exposure, and OR for the sunlight exposed/unexposed groups and their $95 \% \mathrm{CI}$ were presented or the frequency of AMD in the exposed/unexposed group was available in the paper. Case reports, comments and experimental study designs in laboratory settings were excluded. A random-effects meta-analysis, followed by subgroup analysis and sensitivity analysis, including a randomeffects meta-regression for study-specific covariates were done. Fourteen studies were identified. Twelve studies identified an increasing risk of AMD with greater sunlight exposure, six of which reported significant risks. The pooled OR was 1.379 (95\% CI 1.091-1.745). The subgroup of nonpopulation-based studies revealed a significant risk (OR $2.018,1.248-3.265, p=0.004)$. The gross domestic product (GDP) per capita $(p=0.048)$, but not the latitude $(p=0.21)$, was identified as a factor that led to heterogeneity according to the meta-regression. The authors have concluded that the epidemiological literature published to date indicates that individuals with more sunlight exposure are at a significantly increased risk of AMD. The OR significantly decreased with increasing GDP per capita. Subgroup analysis suggested latitude might be a covariate that is negatively correlated with the strength of association, although the meta-regression offered insufficient evidence to support it. More studies using larger scales and better methodologies are needed to confirm these findings and identify other potential risk factors for AMD as well as clarify the uncertain relationship between OR strength and latitude.

Gp Capt M.A. Khan* Associate Professor, Department of Ophthalmology, Armed Forces Medical College, Pune-411040, India

Brig V.S. Gurunadh Professor and Head, Department of Ophthalmology, Armed Forces Medical Collage, Pune-411040, India

${ }^{*}$ Corresponding author. E-mail address: mansurophthal@gmail.com (M.A. Khan)

0377-1237/\$ - see front matter http://dx.doi.org/10.1016/j.mjafi.2013.11.003 